This article is an open access publication

Algebra Universalis

\title{
Free medial quandles
}

\author{
Přemysl JedličKa, Agata Pilitowska, and Anna Zamojska-Dzienio
}

\begin{abstract}
This paper gives the construction of free medial quandles as well as free $n$-symmetric medial quandles and free $m$-reductive medial quandles.
\end{abstract}

\section{Introduction}

A binary algebra $(Q, \cdot)$ is called a rack if the following conditions hold, for every $x, y, z \in Q$ :

- $x(y z)=(x y)(x z)$ (we say $Q$ is left distributive),

- the equation $x u=y$ has a unique solution $u \in Q$ (we say $Q$ is a left quasigroup).

An idempotent rack is called a quandle (we say $Q$ is idempotent if $x x=x$ for every $x \in Q)$. A quandle $Q$ is medial if, for every $x, y, u, v \in Q$,

$$
(x y)(u v)=(x u)(y v) .
$$

An important example of a medial quandle is an abelian group $A$ with an operation $*$ defined by $a * b=(1-h)(a)+h(b)$, where $h$ is an automorphism of $A$. This construction is called an affine quandle (or sometimes an Alexander quandle) and denoted by $\operatorname{Aff}(A, h)$. In the literature $[6,7]$, the group $A$ is usually considered to be a $\mathbb{Z}\left[t, t^{-1}\right]$-module, where $t \cdot a=h(a)$, for each $a \in A$. We shall adopt this point of view here as well and we usually write $\operatorname{Aff}(A, r)$ instead, where $r$ is a ring element.

Note that in universal algebra terminology, an algebra is said to be affine if it is polynomially equivalent to a module. A subreduct of an affine algebra is called a quasi-affine algebra, see e.g., [13]. Clearly, affine quandles are quasiaffine algebras.

Medial quandles lie in the intersection of the class of quandles and the class of modes [16]. Recent development in quandle theory is motivated by knot theory (see e.g., [1, 4]). The knot quandle is a very powerful knot invariant. Quandles also have applications in differential geometry [14] and graph theory [3]. Modes are generally idempotent and entropic algebras (algebras with a commutative clone of term operations). Mediality is another name for entropicity in the binary case. For a more detailed history of medial quandles, we refer to $[9]$.

Presented by P. Dehornoy.

Received April 14, 2016; accepted in final form July 15, 2016.

2010 Mathematics Subject Classification: Primary: 08B20; Secondary: 15A78, 20 N02.

Key words and phrases: quandles, medial quandles, binary modes, free algebras. 
Medial quandles do not form a variety of binary algebras, unless we introduce an additional binary operation $\backslash$ and the identities

$$
x \backslash(x * y) \approx y \quad \text { and } \quad x *(x \backslash y) \approx y
$$

to define the left quasigroup property equationally.

The structure of free medial quandles remained open for a long time. There were only results about more general classes, i.e., general free modes were investigated by Stronkowski [18] and general free quandles by Joyce [11] and Stanovský [17]. In [2], racks and quandles were studied under the names LD-quasigroups and LDI-quasigroups, respectively. Among others, the constructions of free racks and free quandles based on free groups were provided [2, Propositions V.1.17 and X.4.8]. Just recently in [5], such free algebras were again described but the characterization does not give any useful information about their structure.

There were also some special cases investigated, like involutory medial quandles, which means medial quandles satisfying additionally $x *(x * y) \approx y$.

Theorem 1.1 ([11, Theorem 10.5]). Let $n \in \mathbb{N}$. Denote by $F$ the subset of the quandle $\operatorname{Aff}\left(\mathbb{Z}^{n},-1\right)$ consisting of those $n$-tuples where at most one coordinate is odd. Then $(F, *)$ is a free $(n+1)$-generated involutory medial quandle over $\{(0, \ldots, 0),(1,0, \ldots, 0),(0,1,0, \ldots, 0), \ldots,(0, \ldots, 0,1)\}$.

Here we generalize the result of Joyce but not directly. We choose the path started in [9] instead and we study certain permutation groups acting on quandles, called displacement groups. It turns out that, in the case of free medial quandles, these groups are free $\mathbb{Z}\left[t, t^{-1}\right]$-modules and we can construct the free medial quandles based on these modules. Another important result is that the free medial quandles embed into affine quandles. This shows that the variety of medial quandles is generated by affine quandles.

Next we focus on two special classes: $n$-symmetric and $m$-reductive medial quandles which play a significant role within the class of finite medial quandles. A quandle $(Q, *)$ is $n$-symmetric if it satisfies the identity

$$
\underbrace{x *(x * \cdots *(x}_{n-\text { times }} * y) \cdots) \approx y .
$$

We construct here free $n$-symmetric medial quandles and we prove that free $n$-symmetric quandles embed into products of affine quandles over modules over Dedekind domains. This is useful especially when studying finite medial quandles since each finite left quasigroup is $n$-symmetric, for some natural number $n$.

A quandle $(Q, *)$ is $m$-reductive if it satisfies the identity

$$
(\cdots(x * \underbrace{y) * \cdots * y) * y}_{m-\text { times }} \approx y .
$$

A quandle is called reductive if it is $m$-reductive, for some $m \in \mathbb{N}$. Reductivity turns out to be a very important notion in the study of medial quandles as 
each finite medial quandle embeds into a product of a reductive quandle and a quasigroup [10].

The paper contents four Sections. In Section 2, we recall and present some facts about general medial quandles. Section 3 contains the main results. Theorem 3.3 gives a description of free medial quandles and Theorem 3.5 a construction of affine quandles into which the free quandles embed. Section 4 is devoted to $n$-symmetric and $m$-reductive free medial quandles. In both cases, the displacement group of the free algebra turns out to be a free $\mathbb{Z}[t] /(f)$ module, for a suitable polynomial $f$. The description of the free quandles in these varieties is analogous to that for general medial quandles.

Note that when studying left quasigroups, important tools are the mappings $L_{e}: x \mapsto e * x$, called the left translations. We use also the right translations $R_{e}: x \mapsto x * e$. The idempotency and the mediality imply that both $L_{e}$ and $R_{e}$ are endomorphisms. The left quasigroup property means that $L_{e}$ is an automorphism.

\section{Preliminaries}

This section recalls some important notions from [9] where the structure of medial quandles was described. Key ingredients are two permutation groups acting on quandles.

Definition 2.1. Let $Q$ be a quandle.

The left multiplication group of $Q$ is the group $\operatorname{LMlt}(Q)=\left\langle L_{x} ; x \in Q\right\rangle$.

The displacement group is the group $\operatorname{Dis}(Q)=\left\langle L_{x} L_{y}^{-1} ; x, y \in Q\right\rangle$.

It was proved in [8, Proposition 2.1] that the actions of both groups on $Q$ have the same orbits. We use, in the sequel, the word orbit plainly without explicitly mentioning the acting groups. The orbit of $Q$ containing $x$ is denoted by $Q x$ and the stabilizer subgroup of $x$ is denoted by $\operatorname{Dis}(Q)_{x}$. For two permutations $\alpha, \beta$, we write $\alpha^{\beta}=\beta \alpha \beta^{-1}$. The commutator is defined by $[\beta, \alpha]=\alpha^{\beta} \alpha^{-1}$. The identity permutation is denoted by 1 .

It is also useful to understand the structure of the displacement group.

Lemma 2.2 ([8, Proposition 2.1]). Let $Q$ be a quandle.

$$
\operatorname{Dis}(Q)=\left\{L_{x_{1}}^{\varepsilon_{1}} L_{x_{2}}^{\varepsilon_{2}} \cdots L_{x_{k}}^{\varepsilon_{k}} \mid x_{i} \in Q, \varepsilon_{i}= \pm 1, \sum \varepsilon_{i}=0\right\} .
$$

From this lemma, we can clearly see that $\operatorname{Dis}(Q)$ is a normal subgroup of $\operatorname{LMlt}(Q)$. Moreover, in our context, the group is commutative.

Proposition 2.3 ([12]). Let $Q$ be a quandle. Then $Q$ is medial if and only if $\operatorname{Dis}(Q)$ is abelian. 
Since $\operatorname{Dis}(Q)$ is abelian, conjugations by elements from the same coset of $\operatorname{Dis}(Q)$ yield the same results.

Lemma 2.4. Let $Q$ be a medial quandle. Let $\alpha \in \operatorname{Dis}(Q)$ and $x, y \in Q$. Then $\alpha^{L_{x}}=\alpha^{L_{y}}$.

Proof. $\alpha^{L_{x}}=L_{x} \alpha L_{x}^{-1}=L_{x} \alpha L_{x}^{-1} L_{y} L_{y}^{-1}=L_{x} L_{x}^{-1} L_{y} \alpha L_{y}^{-1}=\alpha^{L_{y}}$ due to the abelianess of $\operatorname{Dis}(Q)$.

From now on, by writing $\alpha^{L}$, we mean $\alpha^{L_{x}}$ for an arbitrary $x \in Q$, since conjugation does not depend on the element $x$.

It is easy to see that for $\alpha \in \operatorname{Aut}(Q)$ and $x \in Q$, we have $L_{\alpha(x)}=L_{x}^{\alpha}$. In particular, for $\alpha=L_{y}$, we obtain $L_{y * x}=L_{x}^{L_{y}}$. This implies that $\operatorname{LMlt}(Q)$ has only few generators. On the other hand, $\operatorname{Dis}(Q)$, in spite of being a subgroup of $\operatorname{LMlt}(Q)$, has usually more generators than $\operatorname{LMlt}(Q)$.

Proposition 2.5. Let $Q$ be a medial quandle generated by $X \subset Q$ and choose $z \in X$. Then

- the group $\operatorname{LMlt}(Q)$ is generated by $\left\{L_{x} \mid x \in X\right\}$;

- the group $\operatorname{Dis}(Q)$ is generated by $\left\{\left(L_{x} L_{z}^{-1}\right)^{L^{k}} \mid x \in X, k \in \mathbb{Z}\right\}$.

Proof. The generating set for $\operatorname{LMlt}(Q)$ is obtained by induction using $L_{x * y}=$ $L_{x} L_{y} L_{x}^{-1}$ and $L_{x \backslash y}=L_{x}^{-1} L_{y} L_{x}$.

Suppose now $\alpha \in \operatorname{Dis}(Q)$. By Lemma 2.2 and the previous observation, we can suppose $\alpha=L_{x_{1}}^{\varepsilon_{1}} L_{x_{2}}^{\varepsilon_{2}} \cdots L_{x_{n}}^{\varepsilon_{n}}$, where $x_{i} \in X$ and $\varepsilon_{i}= \pm 1$ with $\sum \varepsilon_{i}=0$ for $1 \leq i \leq n$. We prove the claim by an induction on $n$. For $n=2$, the claim is true.

Let the induction hypothesis hold for words of length $\leq n-2$. If $\varepsilon_{1}=\varepsilon_{n}$, then $w=w_{1} w_{2}$ with $w_{i} \in \operatorname{Dis}(Q)$ and we use the induction hypothesis. Let now $\varepsilon_{1}=1$ and $\varepsilon_{n}=-1$. Then $w=L_{x_{1}} w^{\prime} L_{x_{n}}^{-1}$ and $w^{\prime}$, by the induction hypothesis, is a product of elements from $\left\{\left(L_{x} L_{z}^{-1}\right)^{L^{k}} \mid x \in X, k \in \mathbb{Z}\right\}$. But

$$
w=L_{x_{1}} w^{\prime} L_{x_{1}}^{-1} L_{x_{1}} L_{z}^{-1} L_{z} L_{x_{n}}^{-1}=\left(w^{\prime}\right)^{L}\left(L_{x_{1}} L_{z}^{-1}\right)\left(L_{x_{n}} L_{z}^{-1}\right)^{-1},
$$

proving the claim. The argument is similar for $\varepsilon_{1}=-1$ and $\varepsilon_{n}=1$.

This result cannot be much improved - it is shown in Proposition 3.2 that the displacement group of a free medial quandle is not finitely generated.

The abelian group $\operatorname{Dis}(Q)$ can be easily endowed with the structure of a $\mathbb{Z}\left[t, t^{-1}\right]$-module; it suffices to pick an automorphism of $\operatorname{Dis}(Q)$. A natural choice is the inner automorphism $\alpha \mapsto \alpha^{L}$. Hence, from now on, the group $\operatorname{Dis}(Q)$ is treated, depending on the situation, either as a permutation group acting on $Q$ or as an $R$-module, where $R$ is a suitable image of $\mathbb{Z}\left[t, t^{-1}\right]$, with the action of $t$ defined by $\alpha^{t}=\alpha^{L}$. Note that for $f \in \mathbb{Z}\left[t, t^{-1}\right]$, we have $\alpha^{f}=\alpha^{f(L)}$. 
Example 2.6. Let $f=(1-t)^{2}$. Then

$$
\alpha^{f}=\alpha^{1-2 t+t^{2}}=\alpha^{f(L)}=\alpha^{1-2 L+L^{2}}=\alpha\left(\alpha^{L}\right)^{-2} \alpha^{L^{2}} .
$$

It was proved in [9, Proposition 3.2] that for any $x \in Q$, the orbit $Q x$ is affine over $\operatorname{Dis}(Q) / \operatorname{Dis}(Q)_{x}$ and we can naturally identify the sets $Q x$ and $\operatorname{Dis}(Q) / \operatorname{Dis}(Q)_{x}$ by defining the group operation on $Q x$ as

$$
\alpha(x)+\beta(x)=\alpha \beta(x) \quad \text { and } \quad-\alpha(x)=\alpha^{-1}(x) .
$$

The group so defined is denoted by $\operatorname{Orb}_{Q}(x)$ and called the orbit group for $Q x$. Moreover, $\operatorname{Dis}(Q)_{x}$ is a submodule of $\operatorname{Dis}(Q)$ : suppose $\alpha(x)=x$; then $\alpha^{t}(x)=L_{x} \alpha L_{x}^{-1}(x)=x$. This means that $\operatorname{Dis}(Q) / \operatorname{Dis}(Q)_{x}$ is a $\mathbb{Z}\left[t, t^{-1}\right]$ module and we can call $\operatorname{Orb}_{Q}(x)$ the orbit module for $Q x$.

\section{Free medial quandle}

In this section, we present the free medial quandles. Regarding the generating set, we see that in any quandle $Q$, for all $x, y \in Q$, we have $y * x \in Q x$ as well as $y \backslash x \in Q x$. Hence, each orbit has to contain at least one generator.

Lemma 3.1. Let $Q$ be a quandle generated by $X \subset Q$. Then the set $X \cap Q x$ is nonempty for each $x \in Q$.

The following proposition characterizes the free medial quandles. Formally, it is proved to be a sufficient condition only, but we can see from Theorem 3.3 that such an object exists, making the condition necessary too.

Proposition 3.2. Let $F$ be a medial quandle generated by a set $X \subset F$. Choose $z \in X$ arbitrarily. Then $F$ is free over $X$ if the following conditions are satisfied:

(1) each two elements of $X$ lie in different orbits;

(2) $\operatorname{Dis}(F)$ is a free $\mathbb{Z}\left[t, t^{-1}\right]$-module with $\left\{L_{x} L_{z}^{-1} \mid x \in X \backslash\{z\}\right\}$ as a free basis;

(3) the action of $\operatorname{Dis}(F)$ on $F$ is free.

Proof. First, for any $y \in F$, there exists exactly one $x \in X$ and exactly one $\alpha \in \operatorname{Dis}(F)$ such that $y=\alpha(x)$. Indeed, the existence of $x$ comes from Lemma 3.1, and its uniqueness from (1). The uniqueness of $\alpha$ is due to (3).

Let $Q$ be a medial quandle and let $Y \subset Q$. Let $\psi$ be a mapping $X \rightarrow Y$. We prove that $\psi$ can be extended to a homomorphism $\Psi: F \rightarrow Q$. We define first a $\mathbb{Z}\left[t, t^{-1}\right]$-module homomorphism $\Phi: \operatorname{Dis}(F) \rightarrow \operatorname{Dis}(Q)$ on the basis of $\operatorname{Dis}(F)$ by setting $\Phi\left(L_{x} L_{z}^{-1}\right)=L_{\psi(x)} L_{\psi(z)}^{-1}$. Note that $\Phi\left(\alpha^{L}\right)=\Phi\left(\alpha^{t}\right)=\Phi(\alpha)^{t}=$ $\Phi(\alpha)^{L}$. Now set $\Psi(\alpha(x))=\Phi(\alpha)(\psi(x))$ for all $\alpha \in \operatorname{Dis}(F)$ and $x \in X$. 
The mapping $\Psi$ is well defined since every element of $F$ has a unique representation by $\alpha$ and $x$.

$$
\begin{aligned}
& \Psi(\alpha(x)) * \Psi(\beta(y))=\Phi(\alpha)(\psi(x)) * \Phi(\beta)(\psi(y))=L_{\Phi(\alpha)(\psi(x))} \Phi(\beta)(\psi(y)) \\
& \quad=L_{\psi(x)}^{\Phi(\alpha)} \Phi(\beta)(\psi(y))=\Phi(\alpha) L_{\psi(x)} \Phi\left(\alpha^{-1}\right) \Phi(\beta) L_{\psi(x)}^{-1} L_{\psi(x)} L_{\psi(y)}^{-1}(\psi(y)) \\
& =\Phi(\alpha)\left(\Phi\left(\alpha^{-1} \beta\right)\right)^{L} L_{\psi(x)} L_{\psi(y)}^{-1}(\psi(y))=\Psi\left(\alpha\left(\alpha^{-1} \beta\right)^{L} L_{x} L_{y}^{-1}(y)\right) \\
& \quad=\Psi\left(\alpha L_{x} \alpha^{-1} \beta(y)\right)=\Psi\left(L_{\alpha(x)} \beta(y)\right)=\Psi(\alpha(x) * \beta(y)),
\end{aligned}
$$

and $\Psi$ is a homomorphism that extends $\psi$.

In the sequel, we use the following notation. Let $X$ be a set. We choose $z \in X$ arbitrarily and denote by $X^{-}$the set $X \backslash\{z\}$. We often do not specify the element $z$ since we actually rarely need it explicitly. Now let $R$ be a ring and consider the free $R$-module of rank $\left|X^{-}\right|$, i.e., $M=\bigoplus_{x \in X^{-}} R$. We then choose a free basis of $M$, let us say $\left\{e_{i} \mid i \in X^{-}\right\}$, and by defining $e_{z}=0 \in M$, we have defined $e_{i}$ as an element of $M$, for each $i \in X$.

Theorem 3.3. Let $X$ be a set and let $z \in X$. Denote by $X^{-}$the set $X \backslash\{z\}$. Let $M=\bigoplus_{x \in X^{-}} \mathbb{Z}\left[t, t^{-1}\right]$. Let $\left\{e_{i} \mid i \in X^{-}\right\}$be a free basis of $M$. Moreover, let $e_{z}=0 \in M$. Let us denote by $F$ the set $M \times X$ equipped with the operation

$$
(a, i) *(b, j)=\left((1-t) \cdot a+t \cdot b+e_{i}-e_{j}, j\right) .
$$

Then $(F, *)$ is a free medial quandle over $\{(0, i) \mid i \in X\}$.

Proof. Idempotency is evident. Mediality is proved by the observation that

$$
\begin{aligned}
((a, i) *(b, j)) *((c, k) & *(d, n))=\left((1-t)^{2} \cdot a+\left(t-t^{2}\right) \cdot(b+c)\right. \\
& \left.+t^{2} \cdot d+(1-t) \cdot e_{i}+t \cdot\left(e_{j}+e_{k}\right)-(1+t) \cdot e_{n}, n\right) .
\end{aligned}
$$

The left-quasigroup operation is given by the formula

$$
(a, i) \backslash(b, j)=\left(\left(1-t^{-1}\right) \cdot a+t^{-1} \cdot\left(b+e_{j}-e_{i}\right), j\right) .
$$

Hence, $F$ is a medial quandle.

We know now that $F$ is a medial quandle and we want to prove its freeness by Proposition 3.2.

We start with analyzing the structure of $\operatorname{Dis}(F)$.

$$
\begin{aligned}
L_{(a, i)} L_{(b, j)}^{-1}((c, k)) & =(a, i) *((b, j) \backslash(c, k)) \\
& =(a, i) *\left(\left(1-t^{-1}\right) \cdot b+t^{-1} \cdot\left(c+e_{k}-e_{j}\right), k\right) \\
& =\left((1-t) \cdot a+(t-1) \cdot b+c+e_{k}-e_{j}+e_{i}-e_{k}, k\right) \\
& =\left(c+(1-t) \cdot(a-b)+e_{i}-e_{j}, k\right) .
\end{aligned}
$$

In particular, $L_{(0, i)} L_{(0, z)}^{-1}((c, k))=\left(c+e_{i}, k\right)$. Now we prove by induction that $\left(L_{(0, i)} L_{(0, z)}^{-1}\right)^{L^{n}}((c, j))=\left(c+t^{n} \cdot e_{i}, j\right)$ for each $i \in X^{-}, j \in X$, and $n \in \mathbb{Z}$. 
The case $n=0$ was already proved. Now suppose $n>0$.

$$
\begin{aligned}
\left(L_{(0, i)} L_{(0, z)}^{-1}\right)^{L^{n}}((c, j)) & =L_{(0, z)}\left(L_{(0, i)} L_{(0, z)}^{-1}\right)^{L^{n-1}} L_{(0, z)}^{-1}((c, j)) \\
& =L_{(0, z)}\left(L_{(0, i)} L_{(0, z)}^{-1}\right)^{L^{n-1}}\left(\left(t^{-1} \cdot\left(c+e_{j}\right), j\right)\right) \\
& =L_{(0, z)}\left(\left(t^{-1} \cdot\left(c+e_{j}\right)+t^{n-1} \cdot e_{i}, j\right)\right)=\left(c+t^{n} \cdot e_{i}, j\right) .
\end{aligned}
$$

The case $n<0$ is analogous. Moreover, from this we see that

$$
\left(L_{(0, i)} L_{(0, z)}^{-1}\right)^{f}((c, j))=\left(c+f \cdot e_{i}, j\right),
$$

for any $c \in M, f \in \mathbb{Z}\left[t, t^{-1}\right]$, and $i, j \in X$.

Let $\left(\left(f_{i}\right)_{i \in X^{-}}, j\right)$ be in $F$. We now prove that this element lies in the subquandle generated by $\{(0, i) \mid i \in X\}$. But it is not difficult to see that

$$
\left(\left(f_{i}\right)_{i \in X^{-}}, j\right)=\prod_{i \in X^{-}}\left(L_{(0, i)} L_{(0, z)}^{-1}\right)^{f_{i}}((0, j)) .
$$

The product is finite since only finitely many $f_{i}$ are non-zero, and hence $\langle\{(0, i) \mid i \in X\}\rangle=F$. Moreover, we see that different generators lie in different orbits.

Since the set $\left\{\left(L_{(0, i)} L_{(0, z)}^{-1}\right)^{L^{n}} \mid i \in X, n \in \mathbb{Z}\right\}$ generates $\operatorname{Dis}(F)$ due to Proposition 2.5 , we see that $\operatorname{Dis}(F)$ acts freely on every orbit of $F$. That means also that $\operatorname{Dis}(F)$ is isomorphic to $M$ and $\left\{L_{(0, i)} L_{(0, z)} \mid i \in X^{-}\right\}$is clearly its free basis. According to Proposition 3.2, $F$ is free over $\{(0, i) \mid i \in X\}$.

In [9], the structure of medial quandles was represented using a heterogeneous structure called the indecomposable affine mesh. We do not recall the definition here as it is not needed, we just remark that the free medial quandle now constructed is the sum of the affine mesh

$$
\left(\left(\bigoplus_{x \in X^{-}} \mathbb{Z}\left[t, t^{-1}\right]\right)_{i \in X} ;(1-t)_{i, j \in X} ;\left(e_{i}-e_{j}\right)_{i, j \in X}\right) .
$$

Recall that subquandles of affine quandles are quasi-affine. Every (both sided) cancellative medial quandle is quasi-affine - to see this we can either use a result by Kearnes [13] for idempotent cancellative algebras having a central binary operation or a result by Romanowska and Smith (see e.g., [16]) for cancellative modes. Nevertheless, a direct proof is simple.

Proposition 3.4. Let $Q$ be a cancellative medial quandle. Then $Q$ embeds into any of its orbits.

Proof. $R_{x}$ is an endomorphism of $Q$ for each $x \in Q$. The right cancellativity ensures that $R_{x}$ is injective. Hence, for each $x \in Q, R_{x}$ embeds $Q$ into $Q x$.

The free medial quandle that we have constructed is cancellative, and therefore it can be represented as a subquandle of an affine quandle.

Theorem 3.5. Let $X$ be a set. The free medial quandle over $X$ is isomorphic to a subquandle of the affine quandle $M=\operatorname{Aff}\left(\bigoplus_{x \in X^{-}} \mathbb{Z}\left[t, t^{-1}\right], t\right)$. 
Proof. Let $\Lambda: \bigoplus_{x \in X^{-}} \mathbb{Z}\left[t, t^{-1}\right] \rightarrow \bigoplus_{x \in X^{-}} \mathbb{Z}$ be the group homomorphism induced by the evaluation homomorphism $\mathbb{Z}\left[t, t^{-1}\right] \rightarrow \mathbb{Z}$, where $t \mapsto 1$. Denote $Q=\left\{a \in M \mid \Lambda(a)=e_{i}\right.$, for some $\left.i \in X\right\}$. Then $Q$ is a subquandle of $M$ since

$$
\Lambda\left(L_{a}(b)\right)=\Lambda((1-t) \cdot a+t \cdot b)=0 \cdot \Lambda(a)+1 \cdot \Lambda(b)=\Lambda(b),
$$

and analogously, $\Lambda\left(L_{a}^{-1}(b)\right)=\Lambda(b)$. We shall prove that $Q$ is a free quandle over $\left\{e_{x} \mid x \in X\right\}$.

Take the quandle $F$ from Theorem 3.3. Note that the orbit $F(0, z)$ is isomorphic to $M$ through the bijection $(a, z) \mapsto a$. Now consider the embedding $R_{(0, z)}: F \rightarrow F(0, z)$. Clearly, $R_{(0, z)}((0, i))=\left(e_{i}, 0\right)$. Therefore, the subquandle of $M$ generated by $Y=\left\{e_{x} \mid x \in X\right\}$ is free.

The only thing left to prove is to show that $Q=\langle Y\rangle$. Clearly, $Y \subset Q$ and $Q$ is a subquandle of $M$, hence $Q \supseteq\langle Y\rangle$.

On the other hand, for $Q \subseteq\langle Y\rangle$, we notice that

$$
Q=\left\{a \in M \mid a \equiv e_{i}(\bmod (1-t)) \text {, for some } i \in X\right\} \text {. }
$$

Moreover, we have $\left(L_{e_{x}} L_{e_{z}}^{-1}\right)^{L^{n}}: Q \rightarrow Q ; u \mapsto u+(1-t) t^{n} \cdot e_{x}$, with an analogous proof as in Theorem 3.3. Then $\left(L_{e_{x}} L_{e_{z}}^{-1}\right)^{f(L)}(u)=u+(1-t) f \cdot e_{x}$ for each $f \in \mathbb{Z}\left[t, t^{-1}\right]$. Now, for each element $a \in Q, a=e_{i}+(1-t) g$ for some $i \in X$ and $g=\left(g_{x}\right)_{x \in X^{-}} \in M$. Hence, we have that $a=\prod_{x \in X^{-}}\left(L_{e_{x}} L_{e_{z}}^{-1}\right)^{g_{x}(L)}\left(e_{i}\right)$. Therefore, $a \in\langle Y\rangle$.

Example 3.6. We describe now the free medial quandle on three generators. Let $X=\{0,1,2\}$; let $e_{1}=(1,0), e_{2}=(0,1)$, and $e_{0}=(0,0)$. Now $M=\operatorname{Aff}\left(\mathbb{Z}\left[t, t^{-1}\right] \times \mathbb{Z}\left[t, t^{-1}\right], t\right)$ and $\Lambda: \mathbb{Z}\left[t, t^{-1}\right] \times \mathbb{Z}\left[t, t^{-1}\right] \rightarrow \mathbb{Z}^{2}$ is the group homomorphism induced by the evaluation homomorphism $\mathbb{Z}\left[t, t^{-1}\right] \rightarrow \mathbb{Z}$, where $t \mapsto 1$. Denote $Q=\{a \in M \mid \Lambda(a) \in\{(0,0),(1,0),(0,1)\}\}$. By Theorem 3.5, $Q$ is a subquandle of $M$ and it is the free quandle generated by the set $\{(0,0),(1,0),(0,1)\}$. For example, the element $a=\left(1-t, 1+t-t^{2}\right)$ lies in $Q$ since $\Lambda(a)=(0,1)$. Now $a$ can be represented as

$$
\begin{aligned}
(0,1) & +(1-t)(1, t)=(0,1)+(1-t) \cdot(1,0)+(1-t) t \cdot(0,1) \\
& =\left(L_{(1,0)} L_{(0,0)}^{-1}\right)\left(L_{(0,1)} L_{(0,0)}^{-1}\right)^{L}(0,1)=\left(L_{e_{1}} L_{e_{0}}^{-1}\right)\left(L_{e_{2}} L_{e_{0}}^{-1}\right)^{L}\left(e_{2}\right) .
\end{aligned}
$$

\section{Free quandles in subvarieties}

In this section, we study free $n$-symmetric and free $m$-reductive medial quandles. Both types of varieties have a similar property: they can be characterized by an identity on $\operatorname{Dis}(Q)$.

Definition 4.1. Let $I \subset \mathbb{Z}\left[t, t^{-1}\right]$. We say that a medial quandle $Q$ is an $I$-quandle if $\alpha^{f}=1$ for each $\alpha \in \operatorname{Dis}(Q)$ and $f \in I$. 
If $I$ is an ideal of $\mathbb{Z}\left[t, t^{-1}\right]$ and a $\mathbb{Z}\left[t, t^{-1}\right]$-module $M$ satisfies the identity $f \cdot a=0$ for each $a \in M$ and $f \in I$, then $M$ can be viewed as a module over $\mathbb{Z}\left[t, t^{-1}\right] / I$.

In our context, the set $I$ shall usually be a principal ideal, that means $I=$ $(f)$ for some $f \in \mathbb{Z}\left[t, t^{-1}\right]$. We then write that $Q$ is an $f$-quandle, rather than $\{f\}$-quandle or $(f)$-quandle. If, moreover, $f=\sum_{r=0}^{n} c_{r} t^{r}$ and the coefficient $c_{0}$ is invertible, then $\mathbb{Z}\left[t, t^{-1}\right] / f \cong \mathbb{Z}[t] / f$ since $t^{-1} \equiv-\left(\sum_{r=1}^{n} c_{r} t^{r-1}\right)$. $c_{0}^{-1}(\bmod f)$. We use these remarks since working with the $\operatorname{ring} \mathbb{Z}[t] / f$ is often easier than working with the ring $\mathbb{Z}\left[t, t^{-1}\right]$.

We prepared the framework of $I$-quandles to work with symmetric and reductive medial quandles at once. First note that if $Q$ is an $I$-quandle, then clearly $\operatorname{Orb}_{Q}(x)^{I}=\left\{\alpha^{f}(x) \mid \alpha \in \operatorname{Dis}(Q), f \in I\right\}=\{x\}$. On the other hand, let $\alpha^{f}(x)=x$ for arbitrary $\alpha \in \operatorname{Dis}(Q)$ with $f \in I$ and each $x \in Q$. Hence, the action of $\alpha^{f}$ on $Q$ is trivial and this means $\alpha^{f}=1$ since $\operatorname{Dis}(Q)$ is faithful. This immediately gives the following lemma.

Lemma 4.2. Let $Q$ be a medial quandle and let $I \subset \mathbb{Z}\left[t, t^{-1}\right]$. Then $Q$ is an $I$-quandle if and only if $\operatorname{Orb}_{Q}(x)^{I}=\{x\}$ for each $x \in Q$.

Recall that a quandle $Q$ is $n$-symmetric if $L_{x}^{n}=1$ for each $x \in Q$.

Proposition 4.3. A medial quandle $Q$ is n-symmetric if and only if $Q$ is a $\left(\sum_{r=0}^{n-1} t^{r}\right)$-quandle.

Proof. According to [9, Proposition 7.2], a medial quandle $Q$ is $n$-symmetric if and only if for each $x \in Q, \sum_{r=0}^{n-1}(1-\varphi)^{r} \alpha(x)=x$, where $\varphi: Q x \rightarrow Q x$ is defined by $\alpha(x) \mapsto[\alpha, L](x)$.

Then $(1-\varphi)(\alpha(x))=\alpha(x)-[\alpha, L](x)=\alpha[L, \alpha](x)=\alpha^{L}(x)=\alpha^{t}(x)$, and therefore $\alpha^{\sum_{r=0}^{n-1} t^{r}}(x)=x$ for each $x \in Q$. Hence, according to Lemma 4.2, $Q$ is $n$-symmetric if and only if it is a $\left(\sum_{r=0}^{n-1} t^{r}\right)$-quandle.

Recall that a quandle $Q$ is $m$-reductive if $R_{x}^{m}(y)=x$ for all $x, y \in Q$.

Proposition 4.4. A medial quandle $Q$ is m-reductive if and only if it is a $(1-t)^{m-1}$-quandle.

Proof. According to [9, Proposition 6.2], a medial quandle $Q$ is $m$-reductive if and only if $\varphi^{m-1} \alpha(x)=x$ for each $x \in Q$, where $\varphi: Q x \rightarrow Q x$ is defined by $\alpha(x) \mapsto[\alpha, L](x)$.

Then $\varphi(\alpha(x))=[\alpha, L](x)=\alpha\left(\alpha^{-1}\right)^{L}(x)=\alpha^{(1-t)}(x)$, so $\alpha^{(1-t)^{m-1}}(x)=x$ for each $x$. Hence, according to Lemma $4.2, Q$ is $m$-reductive if and only if it is a $(1-t)^{m-1}$-quandle.

Both mentioned polynomials, i.e., $\sum_{r=0}^{n-1} t^{r}$ and $(1-t)^{m-1}$, have the property that the leading as well as the absolute coefficients are invertible.

In this case, not only can $\operatorname{Dis}(Q)$ be treated as a $\mathbb{Z}[t] / f$-module, but it has fewer generators even as a group. 
Proposition 4.5. Let $f=\sum_{r=0}^{s} c_{r} t^{r}$ be a polynomial with $c_{0}$ and $c_{k}$ invertible and let $Q$ be an $f$-quandle generated by $X \subset Q$. Let $z \in X$ be an arbitrary element. Then $\operatorname{Dis}(Q)$ is generated by

$$
\left\{\left(L_{x} L_{z}^{-1}\right)^{L^{r}} \mid x \in X \backslash\{z\} \text { and } 0 \leq r<s\right\} .
$$

Proof. By Proposition 2.5, the group $\operatorname{Dis}(Q)$ is generated by $\left(L_{x} L_{z}^{-1}\right)^{L^{k}}$ for $x \in X \backslash\{z\}$ and $k \in \mathbb{Z}$. But now

$$
\begin{aligned}
\left(L_{x} L_{z}^{-1}\right)^{L^{-1}} & =\left(L_{x} L_{z}^{-1}\right)^{t^{-1}}=\left(L_{x} L_{z}^{-1}\right)^{-\left(\sum_{r=0}^{s-1} c_{r+1} t^{r}\right) c_{0}^{-1}} \\
& =\prod_{r=0}^{s-1}\left(\left(L_{x} L_{z}^{-1}\right)^{L^{r}}\right)^{-c_{r+1} c_{0}^{-1}} \\
\left(L_{x} L_{z}^{-1}\right)^{L^{s}} & =\left(L_{x} L_{z}^{-1}\right)^{t^{s}}=\left(L_{x} L_{z}^{-1}\right)^{-\left(\sum_{r=0}^{s-1} c_{r} t^{r}\right) c_{s}^{-1}} \\
& =\prod_{r=0}^{s-1}\left(\left(L_{x} L_{z}^{-1}\right)^{L^{r}}\right)^{-c_{r} c_{s}^{-1}} .
\end{aligned}
$$

Similarly for all $\left(L_{x} L_{z}^{-1}\right)^{L^{r}}$, where $r<-1$ or $r>s$.

The structure of free medial $f$-quandles can be described exactly in the same way as the structure of general free medial quandles.

Proposition 4.6. Let $f \in \mathbb{Z}[t]$ be a polynomial with the leading and the absolute coefficients invertible. Let $F$ be an $f$-quandle generated by a set $X \subset F$. Choose $z \in X$ arbitrarily. Then $F$ is a free $f$-quandle over $X$ if the following conditions are satisfied:

(1) each two elements of $X$ lie in different orbits;

(2) $\operatorname{Dis}(F)$ is a free $\mathbb{Z}[t] / f$-module with $\left\{L_{x} L_{z}^{-1} \mid x \in X \backslash\{z\}\right\}$ as a free basis;

(3) the action of $\operatorname{Dis}(F)$ on $F$ is free.

Proof. The proof is nearly the same as the proof of Proposition 3.2. The only difference is that displacement groups appearing here are $\mathbb{Z}[t] / f$-modules.

Theorem 4.7. Let $f \in \mathbb{Z}[t]$ be a polynomial with the leading and the absolute coefficients invertible. Let $X$ be a set. Let $M=\bigoplus_{s \in X^{-}} \mathbb{Z}[t] / f$. Let us denote by $F$ the set $M \times X$ equipped with the operation

$$
(a, i) *(b, j)=\left((1-t) \cdot a+t \cdot b+e_{i}-e_{j}, j\right) .
$$

Then $(F, *)$ is a free $f$-quandle over $\{(0, i) \mid i \in X\}$.

Proof. The proof is nearly the same as of Theorem 3.3, with the usage of Proposition 4.6. The only thing to show is that $\alpha^{f}=1$ for all $\alpha \in \operatorname{Dis}(F)$. But this follows from $\operatorname{Dis}(F) \cong M$.

Non-trivial reductive medial quandles are never right-cancellative since the multiplication by $(1-t)$ is not injective. On the other hand, free $n$-symmetric quandles are cancellative and we can embed them in their orbits. Moreover, the polynomial $\sum t^{r}$ is a product of cyclotomic polynomials. 
Theorem 4.8. Let $X$ be a set. Let $n \in \mathbb{N}$ and let $\sum_{r=0}^{n-1} t^{r}=\prod_{j \in \mathcal{J}} f_{j}$, where $f_{j}$ are irreducible in $\mathbb{Z}[t]$ and $M_{j}=\operatorname{Aff}\left(\bigoplus_{x \in X^{-}} \mathbb{Z}[t] / f_{j}, t\right)$ for each $j \in \mathcal{J}$. Each free n-symmetric medial quandle is isomorphic to the subquandle of $\prod_{j \in \mathcal{J}} M_{j}$ generated by $\left\{\left(e_{i}\right)_{j \in \mathcal{J}} \mid i \in X\right\}$.

Proof. We show that the subquandle

$$
Q=\left\{\left(a_{j}\right)_{j \in \mathcal{J}} \mid \exists i \in X \forall j \in \mathcal{J} a_{j} \equiv e_{i}(\bmod (1-t))\right\}
$$

of $\prod_{j \in \mathcal{J}} M_{j}$ is a free $n$-symmetric medial quandle over $\left\{\left(e_{i}, e_{i}, \ldots, e_{i}\right) \mid i \in X\right\}$.

Let $f=\sum_{r=0}^{n-1} t^{r}$. Then $t^{n}-1=(t-1) f=(t-1) \prod_{j \in \mathcal{J}} f_{j}$, and all the polynomials $f_{j}$ are pairwise different. Therefore, by the Chinese remainder theorem, $\mathbb{Z}[t] / f \cong \bigoplus_{j \in \mathcal{J}} \mathbb{Z}[t] / f_{j}$. The rest is the same as in Theorem 3.5.

Example 4.9. Consider $n=2$, i.e., involutory medial quandles. By Theorem 4.8 , and since $\mathbb{Z}[t] /(t+1) \cong \mathbb{Z}$ and $t \equiv-1(\bmod (t+1))$, so the free $|X|$-generated involutory medial quandle is isomorphic to the subquandle of $\operatorname{Aff}\left(\bigoplus_{x \in X^{-}} \mathbb{Z},-1\right)$ that consists of those $\left|X^{-}\right|$-tuples congruent to some $e_{i}$ modulo 2. This confirms the result of Joyce [11, Theorem 10.5].

Theorem 4.8 can be reformulated as follows: let $\zeta_{k}$ be a primitive $k$-th root of unity in $\mathbb{C}$. It is well known that $\mathbb{Z}[t] /\left(1+t+\cdots+t^{n-1}\right) \cong \prod_{k \mid n, k>1} \mathbb{Z}\left[\zeta_{k}\right]$. Hence, the free $|X|$-generated $n$-symmetric quandle is the subquandle of

$$
\prod_{k \mid n, k>1} \operatorname{Aff}\left(\bigoplus_{x \in X^{-}} \mathbb{Z}\left[\zeta_{k}\right], \zeta_{k}\right)
$$

generated by $\left\{\left(e_{i}, e_{i}, \ldots, e_{i}\right) \mid i \in X\right\}$.

Example 4.10. For $|X|=2$, the free 2 -generated $n$-symmetric medial quandle $F$ is the subquandle of $\prod_{k \mid n, k>1} \operatorname{Aff}\left(\mathbb{Z}\left[\zeta_{k}\right], \zeta_{k}\right)$ generated by $(0, \ldots, 0)$ and $(1, \ldots, 1)$, and $F$ consists of the tuples $\left(a_{k}\right)_{k \mid n, k>1}$ where $a_{k} \equiv 0\left(\bmod \left(1-\zeta_{k}\right)\right)$ for all $k \mid n$ with $k>1$, or $a_{k} \equiv 1\left(\bmod \left(1-\zeta_{k}\right)\right)$ for all $k \mid n$ with $k>1$.

Every finite quandle is $n$-symmetric for some $n$. For studying finite medial quandles, it is nice to know that we need not always consider $\mathbb{Z}\left[t, t^{-1}\right]$-modules but we can sometimes focus on nicer rings, or even domains.

Corollary 4.11. Let $n \in \mathbb{N}$. The variety of $n$-symmetric medial quandles is generated by quandles that are polynomially equivalent to modules over Dedekind domains.

Proof. From [19], $\mathbb{Z}\left[\zeta_{k}\right]$ is a Dedekind domain for each $k$. Free $n$-symmetric quandles embed into products of $\operatorname{Aff}\left(\bigoplus_{x \in X^{-}} \mathbb{Z}\left[\zeta_{k}\right], \zeta_{k}\right)$. Each of these affine quandles embeds into any of its orbits, i.e., into a module over $\mathbb{Z}\left[\zeta_{k}\right]$.

Note that by applying our idea of $I$-quandles, one obtains the description of free $n$-symmetric $m$-reductive medial quandles if we consider $I=$ $\left\{\sum_{r=0}^{n-1} t^{r},(1-t)^{m-1}\right\}$. In particular, the free 2-reductive $n$-symmetric medial quandle over $\mathrm{X}$ is isomorphic to $\bigoplus_{x \in X^{-}} \mathbb{Z}_{n} \times X$ with the operation $(a, i) *(b, j)=\left(b+e_{i}-e_{j}, j\right),[15$, Proposition 2.4]. 


\section{REFERENCES}

[1] Carter, J.S.: A survey of quandle ideas. In: Introductory lectures on knot theory. Ser. Knots Everything, vol. 46, pp. 22-53. World Sci. Publ., Hackensack (2012)

[2] Dehornoy, P.: Braids and self-distributivity. Progress in Maths, vol. 192. Birkhaüser (1999)

[3] Denes, J., Keedwell, A.D.: On P-quasigroups and decompositions of complete undirected graphs. J. Combin. Theory Ser. B 13, 270-275 (1972)

[4] Elhamdadi, M., Nelson, S.: Quandles: An introduction to the algebra of knots. American Mathematical Society, Providence (2015)

[5] Farinati, M., Guccione, J.A., Guccione, J.J.: The homology of free racks and quandles. Comm. Algebra 42, 3593-3606 (2014)

[6] Hou, X.: Automorphism groups of Alexander quandles. J. Algebra 344, 373-385 (2011)

[7] Hou, X.: Finite modules over $\mathbb{Z}\left[t, t^{-1}\right]$. J. Knot Theory Ramifications 21, 1250079, 28 pp. (2012)

[8] Hulpke, A., Stanovský, D., Vojtěchovský P.: Connected quandles and transitive groups. J. Pure Appl. Algebra 220, 735-758 (2016)

[9] Jedlička, P., Pilitowska, A., Stanovský, D., Zamojska-Dzienio, A.: The structure of medial quandles. J. Algebra 443, 300-334 (2015)

[10] Jedlička, P., Pilitowska, A., Zamojska-Dzienio, A.: Subdirectly irreducible medial quandles (2016, preprint). http://arxiv.org/abs/1511.06529

[11] Joyce, D.: Classifying invariant of knots, the knot quandle. J. Pure Applied Algebra 23, 37-65 (1982)

[12] Joyce, D.: Simple quandles. J. Algebra 79, 307-318 (1982)

[13] Kearnes, K.: A quasi-affine representation. Internat. J. Algebra Comput. 5, 673-702 (1995)

[14] Loos, O.: Symmetric spaces. W.A. Benjamin, New York (1969)

[15] Romanowska, A., Roszkowska, B.: Representations of $n$-cyclic groupoids. Algebra Universalis 26, 7-15 (1989)

[16] Romanowska, A., Smith, J.D.H.: Modes. World Scientific, Singapore (2002)

[17] Stanovský, D.: Left symmetric left distributive operations on a group. Algebra Universalis 54, 97-103 (2005)

[18] Stronkowski, M.M.: On free modes. Comment. Math. Univ. Carolinae 47, 561-568 (2006)

[19] Zariski, O., Samuel, P.: Commutative algebra, vol. I. Springer, Berlin (1975)

\section{PřEMYSL JEDLIČKA}

Department of Mathematics, Faculty of Engineering, Czech University of Life Sciences, Kamýcká 129, 16521 Praha 6, Czech Republic

e-mail: jedlickap@tf.czu.cz

\section{Agata Pilitowska}

Faculty of Mathematics and Information Science, Warsaw University of Technology,

Koszykowa 75, 00-662 Warsaw, Poland

e-mail: apili@mini.pw.edu.pl

\section{Anna Zamojska-DZienio}

Faculty of Mathematics and Information Science, Warsaw University of Technology, Koszykowa 75, 00-662 Warsaw, Poland e-mail: A.Zamojska-Dzienio@mini.pw.edu.pl

Open Access This article is distributed under the terms of the Creative Commons Attribution 4.0 International License (http://creativecommons.org/licenses/by/4.0/), which permits unrestricted use, distribution, and reproduction in any medium, provided you give appropriate credit to the original author(s) and the source, provide a link to the Creative Commons license, and indicate if changes were made. 